Open Access

\title{
The crucial role of PpMYB10.1 in
}

\section{anthocyanin accumulation in peach and relationships between its allelic type and skin color phenotype}

Pham Anh Tuan ${ }^{1}$, Songling Bai ${ }^{1}$, Hideaki Yaegaki ${ }^{1}$, Takayuki Tamura² ${ }^{2}$ Seisuke Hihara², Takaya Moriguchi ${ }^{*}$ and Kenji Oda ${ }^{3^{*}}$

\begin{abstract}
Background: Red coloration of fruit skin is one of the most important traits in peach (Prunus persica), and it is mainly due to the accumulation of anthocyanins. Three MYB10 genes, PpMYB10.1, PpMYB10.2, and PpMYB10.3, have been reported as important regulators of red coloration and anthocyanin biosynthesis in peach fruit. In this study, contribution of PpMYB10.1/2/3 to anthocyanin accumulation in the fruit skin was investigated in the Japanese peach cultivars, white-skinned 'Mochizuki' and red-skinned 'Akatsuki'. We then investigated the relationships between allelic type of PPMYB10.1 and skin color phenotype in 23 Japanese peach cultivars for future establishment of DNA-marker.

Results: During the fruit development of 'Mochizuki' and 'Akatsuki', anthocyanin accumulation was observed only in the skin of red 'Akatsuki' fruit in the late ripening stages concomitant with high mRNA levels of the last step gene leading to anthocyanin accumulation, UDP-glucose:flavonoid-3-O-glucosyltransferase (UFGT). This was also correlated with the expression level of PpMYB10.1. Unlike PpMYB10.1, expression levels of PpMYB10.2/3 were low in the skin of both 'Mochizuki' and 'Akatsuki' throughout fruit development. Moreover, only PpMYB10.1 revealed expression levels associated with total anthocyanin accumulation in the leaves and flowers of 'Mochizuki' and 'Akatsuki'. Introduction of PpMYB10.1 into tobacco increased the expression of tobacco UFGT, resulting in higher anthocyanin accumulation and deeper red transgenic tobacco flowers; however, overexpression of PpMYB10.2/3 did not alter anthocyanin content and color of transgenic tobacco flowers when compared with wild-type flowers. Dual-luciferase assay showed that the co-infiltration of PpMYB10.1 with PpbHLH3 significantly increased the activity of PpUFGT promoter. We also found close relationships of two PpMYB10.1 allelic types, MYB10.1-1/MYB10.1-2, with the intensity of red skin coloration.

(Continued on next page)
\end{abstract}

\footnotetext{
*Correspondence: takaya@affrc.go.jp; oda@bio-ribs.com

${ }^{1}$ NARO Institute of Fruit Tree Science, 2-1 Fujimoto, Tsukuba, Ibaraki 305-8605, Japan

${ }^{3}$ Research Institute for Biological Sciences, Okayama Prefectural Technology Center for Agriculture Forestry, and Fisheries, 7549-1 Yoshikawa, Kibi-chou, Okayama 716-1241, Japan

Full list of author information is available at the end of the article
}

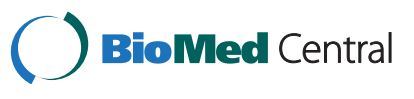

(c) 2015 Tuan et al. Open Access This article is distributed under the terms of the Creative Commons Attribution 4.0 International License (http://creativecommons.org/licenses/by/4.0/), which permits unrestricted use, distribution, and reproduction in any medium, provided you give appropriate credit to the original author(s) and the source, provide a link to the Creative Commons license, and indicate if changes were made. The Creative Commons Public Domain Dedication waiver (http://creativecommons.org/publicdomain/zero/1.0/) applies to the data made available in this article, unless otherwise stated. 
(Continued from previous page)

Conclusion: We showed that PPMYB10.1 is a major regulator of anthocyanin accumulation in red-skinned peach and that it activates PPUFGT transcription. PPMYB10.2/3 may be involved in functions other than anthocyanin accumulation in peach. The peach cultivars having two MYB10.1-2 types resulted in the white skin color. By contrast, those with two MYB10.1-1 or MYB10.1-1/MYB10.1-2 types showed respective red or pale red skin color. These findings contribute to clarifying the molecular mechanisms of anthocyanin accumulation and generating gene-based markers linked to skin color phenotypes.

Keywords: Anthocyanin, Japanese peach cultivars, MYB10 transcription factor, Prunus persica, Skin color, Transgenic tobacco

\section{Background}

Peach (Prunus persica) is an important deciduous fruit, and its total production is ranked as 4th after grape, apple, and pear worldwide. China is the world's leading producer of peach fruit, accounting for about $57 \%$ of the total production. In Japan, peach is ranked $6^{\text {th }}$ in production, after mandarin, apple, pear, persimmon, and grape in 2012. Fruit skin color is one of the most important traits for the commercial value of peach fruit, and it is mainly determined by the content and composition of anthocyanins for red color or carotenoids for yellow color $[1,2]$. With respect to carotenoid accumulation, yellow- and white-skinned types have been found, and the trait is controlled by a single $Y / y$ locus in linkage group $1[3,4]$. Recently, characterization of the $Y / y$ locus has been reported by several research groups; carotenoid cleavage deoxygenase 4 (CCD4) has been identified as a regulator of yellow pigmentation, and loss of function of CCD4 results in the yellow-skinned type [5-9]. In contrast, red coloration of red-skinned peach depends on the accumulation of anthocyanins, which are watersoluble pigments of the flavonoid biosynthetic pathway. The intensity of red coloration is known to show variations depending on cultivars and strains, which suggests that red coloration is genetically controlled. Moreover, anthocyanin accumulation in the skin largely depends on environmental factors such as light and temperature conditions [10-12]. Most Japanese cultivars, including 'Akatsuki', show red skin color when environmental conditions are appropriate, while some Japanese cultivars, such as 'Mochizuki', seldom accumulate anthocyanin; therefore, this type of cultivar is suitable for canned processing. In Japan, red-skinned peach has a generally high market value, so farmers sometimes use the paperbagging treatment for enhancing skin color, although production of white-skinned peach by using red-skinned cultivars (called "Hakuto") has been established in Okayama Prefecture in Japan (http://world.momotaros.com/peach.html).

The molecular mechanism underlying anthocyanin accumulation has been well-characterized in fruit trees [13-15]. Recently, many structural genes involved in the anthocyanin biosynthetic pathway and various transcription factors have been identified and characterized (Fig. 1). Of these, MYB transcription factor genes were often found to be the major determinant of anthocyanin accumulation by acting together with basic helix-loophelix (bHLH) and WD40 proteins (termed the MBW complex) to activate key anthocyanin biosynthetic genes [15-17]. In grape, MYB genes contribute to anthocyanin biosynthesis via expression of UFGT $[18,19]$. In apple, MYBs are involved in the activation of anthocyanin biosynthetic genes, and they regulate the accumulation of anthocyanin in fruit $[20,21]$. In pear, the transcription level of MYB10 in the skin was positively correlated with anthocyanin biosynthetic gene pathway and anthocyanin biosynthesis $[22,23]$. In peach, three MYB10 genes, PpMYB10.1 (Genome Database for Rosaceae accession number: ppa026640m), PpMYB10.2 (ppa016711m), and PpMYB10.3 (ppa020385m), localized in a genomic region of linkage group 3 where the Anther color $(A g)$ trait is located, have been reported as important regulators of anthocyanin biosynthesis in peach fruit [24]. PpMYB10.2 positively regulates the promoter of PpUFGT, which is the only gene that shows a similar expression pattern to that of anthocyanin accumulation in peach skin during fruit development [25]. Rahim et al. [26] showed that the expression levels of $P p M Y B 10.1$ and $P p M Y B 10.3$ correlate with anthocyanin content as well as expression levels of key structural genes in the anthocyanin biosynthetic pathway. Our preliminary study on anthocyanin accumulation using red-skinned cultivars showed high expression levels of PpMYB10.1 but quite low levels of $P p M Y B 10.3$, which may indicate that anthocyanin accumulation in peach skin is dominantly regulated by only PpMYB10.1.

The aim of this study was to evaluate the molecular characterization of the three PpMYB10 genes by using Japanese peach cultivars. We first used two Japanese peach cultivars, white-skinned 'Mochizuki' and redskinned 'Akatsuki', to study the relationship between the transcription levels of $P p M Y B 10.1 / 2 / 3$, anthocyanin biosynthetic genes, and anthocyanin accumulation in fruit skin during fruit development. Next, we analyzed 


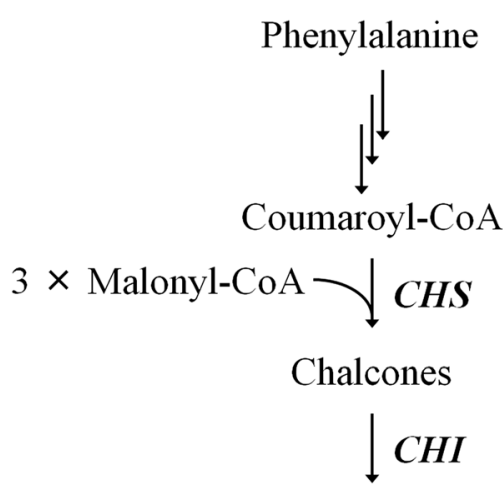

Flavanones

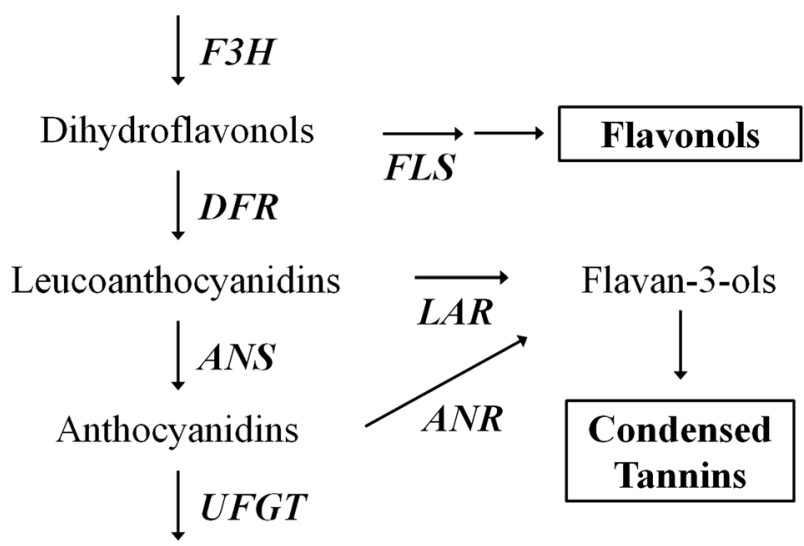

Anthocyanins

Fig. 1 Flavonoid biosynthetic pathway in plants. CHS, Chalcone synthase; CHl, chalcone isomerase; F3H, flavanone 3-hydroxylase; DFR, dihydroflavonol-4reductase; ANS, anthocyanidin synthase; UFGT, UDP-glucose:flavonoid-3-O-glucosyltransferase

overexpression of $P p M Y B 10.1 / 2 / 3$ in tobacco and regulation of PpUFGT promoter activity by PpMYB10.1. Finally, we investigated the intensity of red coloration in the peach skin based on the allelic type of PpMYB10.1.

\section{Results}

Total anthocyanin content and expression analysis of anthocyanin biosynthetic genes in fruit skin of 'Mochizuki' and 'Akatsuki'

The fruit skin of 'Mochizuki' is green in the first four stages and pale-yellow in the ripening stage, stage 5 (Fig. 2a). 'Akatsuki' fruit skin is also green in the early stages and partially or nearly red in stages 4 and 5 , respectively (Fig. 2a). Total anthocyanin content in fruit skin was measured (Fig. 2b). As expected, white-skinned 'Mochizuki' did not show anthocyanin in the skin throughout fruit development. Anthocyanin was also not found at the beginning of 'Akatsuki' fruit development, and it only appeared in stage 4 and increased to a great extent in stage 5 . This is in accordance with the red coloration observed in stages 4 and 5 of 'Akatsuki' skin.
Expression profiles of structural genes involved in anthocyanin biosynthesis were examined using quantitative real-time PCR (qRT-PCR) (Fig. 2c). In general, genes involved in the upstream pathway, including $\mathrm{PpCHS}$, $P p C H I, P p F 3 H$, and $P p D F R$, showed similar expression patterns in the skin of 'Mochizuki' and 'Akatsuki' during fruit development; the expression levels increased from stage 1 , peaked at stage 2 , and then decreased in the last three stages. This is also the expression pattern of $P p A N S$ in the skin of 'Mochizuki', while PpANS revealed the highest expression level in stage 5 for 'Akatsuki'. The mRNA level of PpUFGT was low in 'Mochizuki' skin in all stages, and it was also low in 'Akatsuki' skin in the three early stages and increased in stages 4 and 5 . Although the expression levels of $P p C H S, P p D F R$, and PpANS were higher in the skin in stages 4 and 5 of 'Akatsuki' fruit, only the last step gene that directly leads to anthocyanin accumulation, PpUFGT showed an expression pattern tightly correlated with anthocyanin accumulation in the skin throughout fruit development in 'Mochizuki' and 'Akatsuki'. These results suggest that PpUFGT is the key gene for anthocyanin accumulation 
a

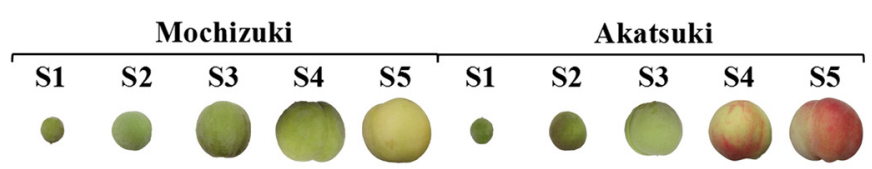

b

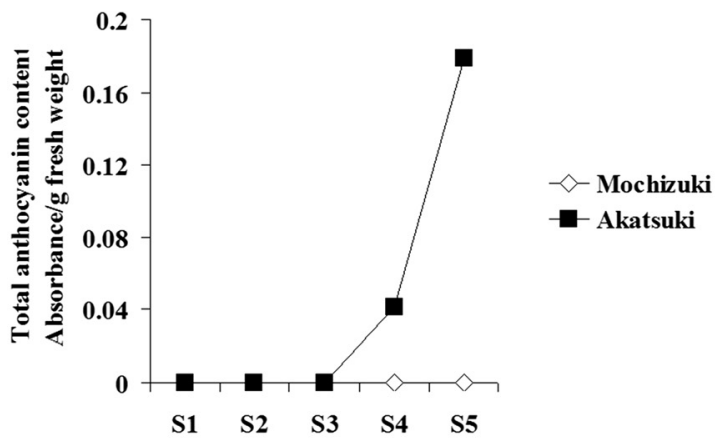

C
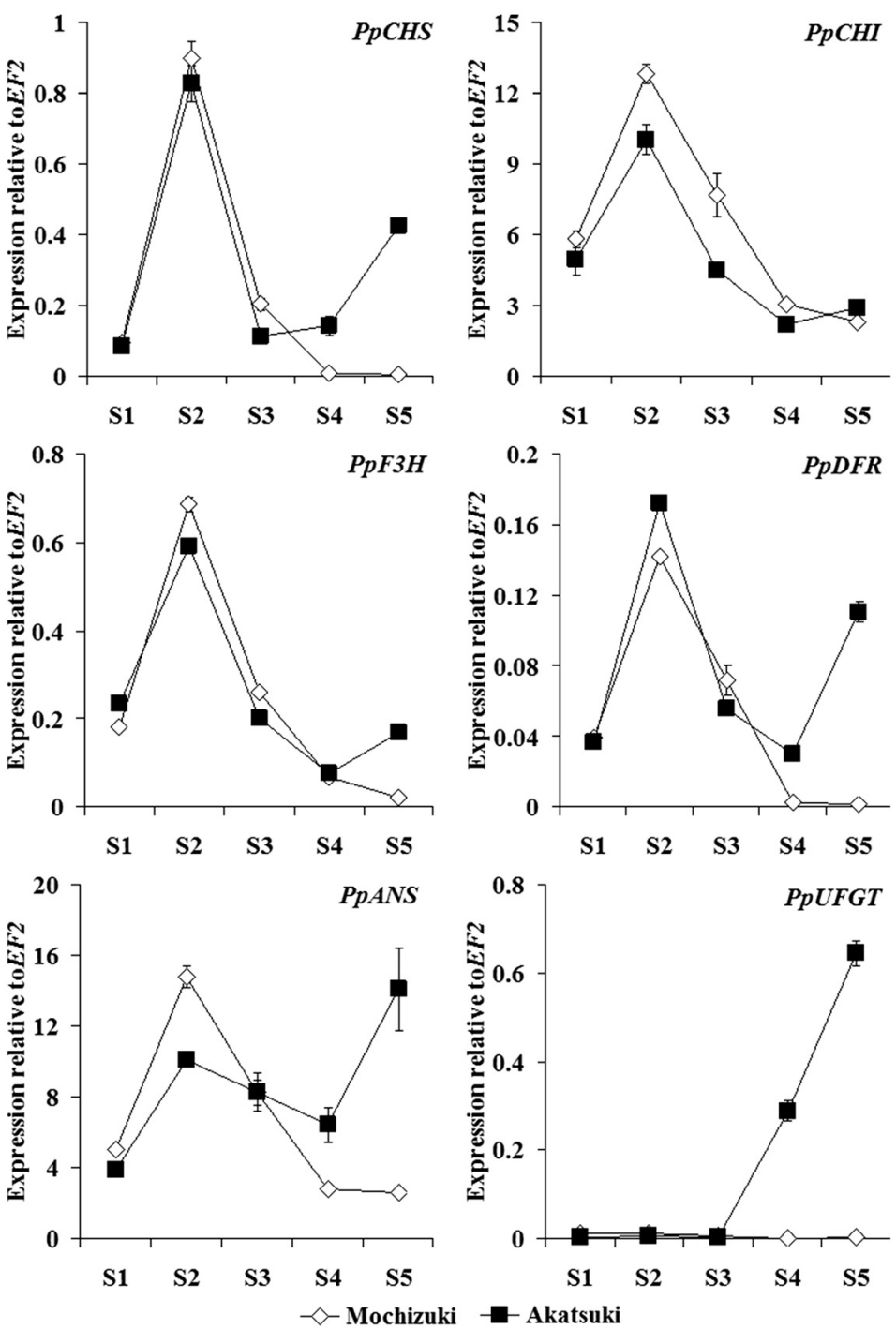

Fig. 2 a Photographs of fruit skin. b Total anthocyanin content. c Expression levels of structural genes involved in the anthocyanin biosynthetic pathway in the skin of 'Mochizuki' and 'Akatsuki' during fruit development. Height of the bars and error bars shows the mean and standard error, respectively, from three independent measurements 
in the skin of 'Mochizuki' and 'Akatsuki' fruit. Therefore, we characterized $M Y B$ genes that could act as a transfactor of PpUFGT.

\section{Expression analysis of PpMYB10.1/2/3 in the skin of 'Mochizuki' and 'Akatsuki' fruit}

PpMYB10.1, PpMYB10.2, and PpMYB10.3 are localized near each other in linkage group 3 . Due to high similarity of the nucleotide sequences among these PpMYB10s (Fig. 3a), qRT-PCR primers for PpMYB10.1/2/3 were manually designed on the basis of divergent nucleotide sequences between them. Real-time PCR products were then carefully tested for specificity by cloning into a pCR2.1-TOPO vector, and seven individual plasmid clones were sequenced to ensure product specificity for each primer set. Expression levels of PpMYB10.1/2/3 were low in the skin during the five developmental stages of 'Mochizuki' fruit (Fig. 3b). In 'Akatsuki' skin, expression levels of $P p M Y B 10.1 / 2 / 3$ were also low at the beginning of fruit development; then, transcription levels of PpMYB10.1 dramatically increased in stages 4 and 5, while expression levels of $P p M Y B 10.2 / 3$ remained low throughout fruit maturation. High mRNA levels of PpMYB10.1 found in stages 4 and 5 were correlated with anthocyanin content and red pigmentation, which were observed only in these two ripening stages of 'Akatsuki' fruit. These results demonstrated that PpMYB10.1 alone is responsible for anthocyanin accumulation in the skin of 'Akatsuki'. To confirm this assumption, we then created transgenic tobacco plants that overexpressed the three PpMYB10 genes.

\section{Characterization of transgenic tobacco plants that overexpressed PpMYB10.1/2/3}

ORFs of PpMYB10.1/2/3 driven by the CaMV 35S promoter were introduced into Nicotiana tabacum SR1 by using Agrobacterium tumefaciens strain LBA4404. Regenerated plants on plates containing $50 \mathrm{mg} / \mathrm{L}$ of kanamycin were examined for the presence of transgenes by using PCR with extracted genomic DNA (Additional file 1: Figure S1a). For each overexpression construct, six independent lines of transgenic plants showing the presence of the corresponding transgene were selected to transfer to the soil and grown under greenhouse conditions. As observed in Fig. 4a, introduction of PpMYB10.1 resulted in a deeper red color in transgenic tobacco flowers when compared with the wild-type tobacco flowers. The capsule skin of transgenic tobacco plants overexpressing PpMYB10.1 also displayed a pale red color, while the capsule skin of wild-type tobacco was green (Additional file 1: Figure S1b). Transgenic tobacco plants transformed with $P p M Y B 10.2 / 3$ showed no coloration differences with respect to flowers when compared with wild-type flowers (Fig. 4a). This color observation reflected that obviously higher anthocyanin accumulation was only found in the flowers of six PpMYB10.1 transgenic tobacco lines (Fig. 4b). To investigate the regulation of branching genes for specific flavonoid groups, such as flavonols and tannins, by transgenes, expression levels of transgenes PpMYB10.1/2/3 and $N$. tabacum FLS, LAR, ANR, and UFGT were analyzed in transgenic tobacco flowers (Additional file 2: Figure S2). The results showed that all PpMYB10.1/2/3 mRNAs were transcribed (Fig. 5a). Moreover, overexpression of PpMYB10.1 substantially upregulated only NtUFGT

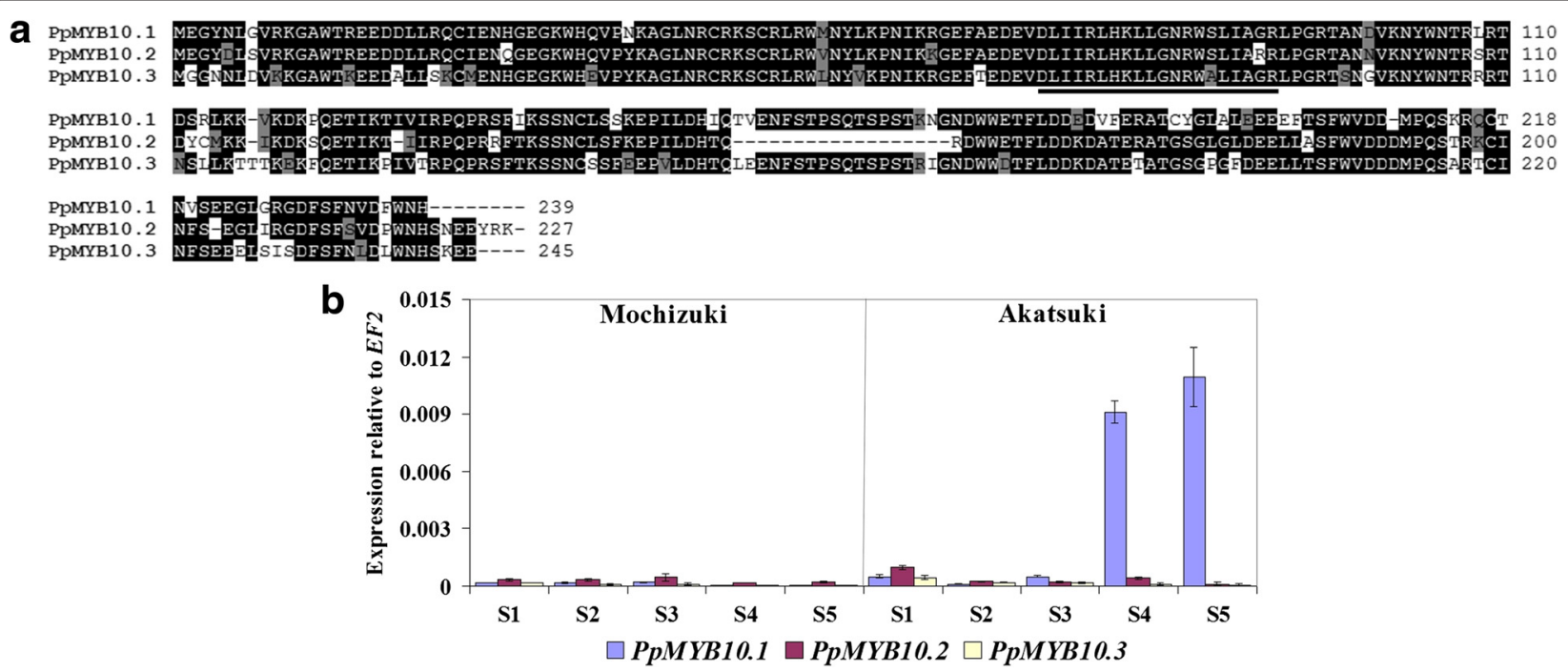

Fig. 3 a Amino acid sequence alignment of PpMYB10.1/2/3. Sequences were retrieved from Genome Database for Rosaceae website (https:// www.rosaceae.org/species/prunus/prunus_persica) in which red peach cultivar 'Lovell' was used to sequence. The solid underline is the R/B-like bHLH binding motif ([DE]L $\left.x_{2}[R K] x_{3} L x_{6} L x_{3} R\right)$. $\mathbf{b}$ Expression levels of PpMYB10.1/2/3 in the skin of 'Mochizuki' and 'Akatsuki' during fruit development. Height of the bars and error bars shows the mean and standard error, respectively, from three independent measurements 


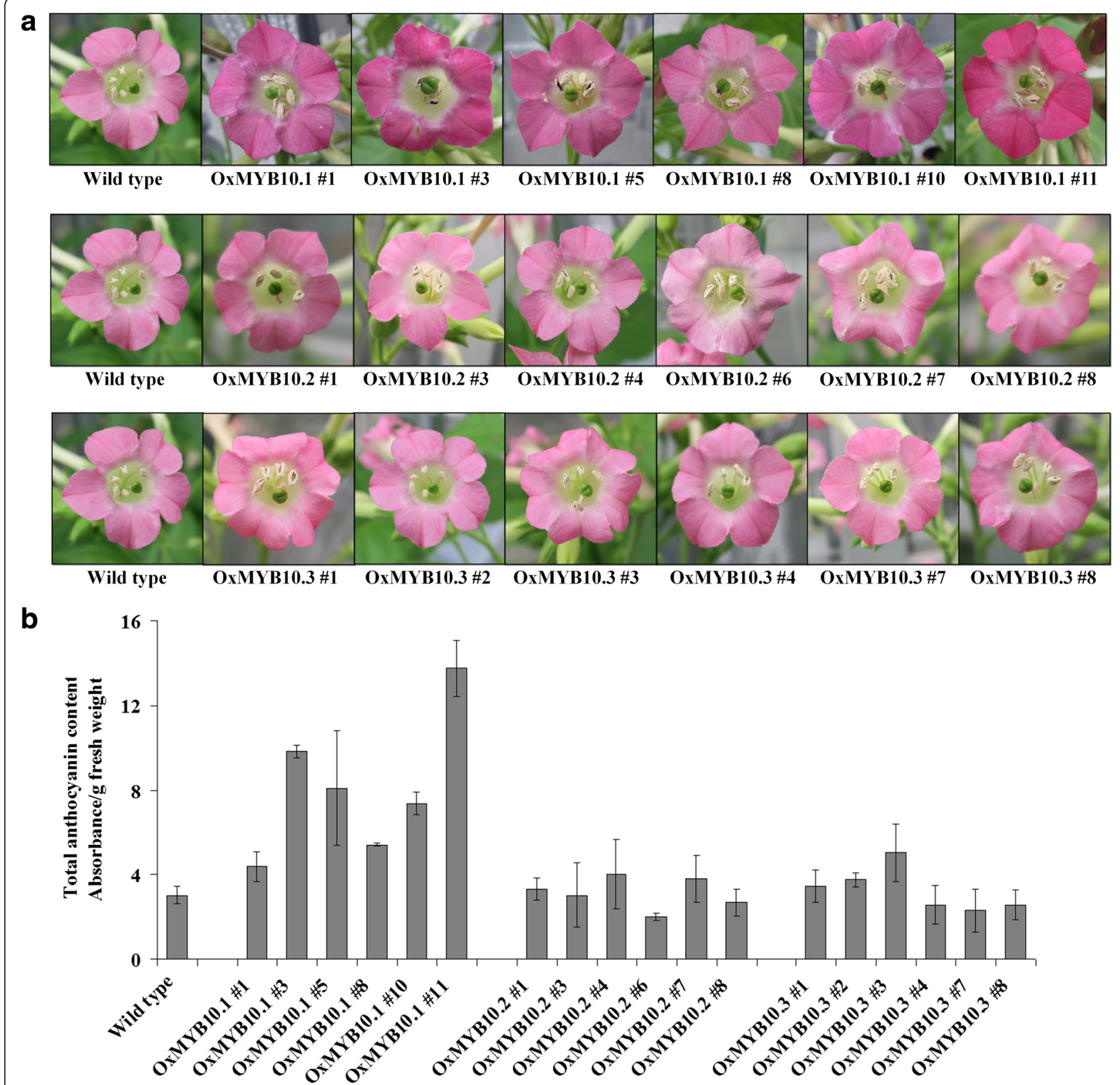

Fig. 4 Photographs (a) and total anthocyanin content (b) of transgenic tobacco flowers overexpressing PpMYB10.1/2/3. Height of the bars and error bars shows the mean and standard error, respectively, from three independent measurements

expression, and overexpression of PpMYB10.2/3 did not markedly alter the transcription of all four examined genes in transgenic tobacco flowers (Fig. 5b, Additional file 2: Figure S2). In addition, expression level of NtUFGT was consistent with the expression level of $P p M Y B 10.1$ transgene in six independent transgenic lines (Fig. 5). Taken together, only PpMYB10.1 can activate tobacco NtUFGT, resulting in higher anthocyanin accumulation and deeper red color in transgenic tobacco flowers. Then, what are the functions of PpMYB10.2 and PpMYB10.3? To confirm this, we analyzed expressions in leaves and flowers of 'Mochizuki' and 'Akatsuki'.

Total anthocyanin content and expression analysis of PpMYB10.1/2/3 in leaves and flowers of 'Mochizuki' and 'Akatsuki'

Leaves of both 'Mochizuki' and 'Akatsuki' are green and showed no anthocyanin accumulation and very low PpUFGT transcription (Fig. 6a, b). PpMYB10.1 and PpMYB10.3 were also poorly transcribed, but a high expression level of PpMYB10.2 was found in the leaves of 


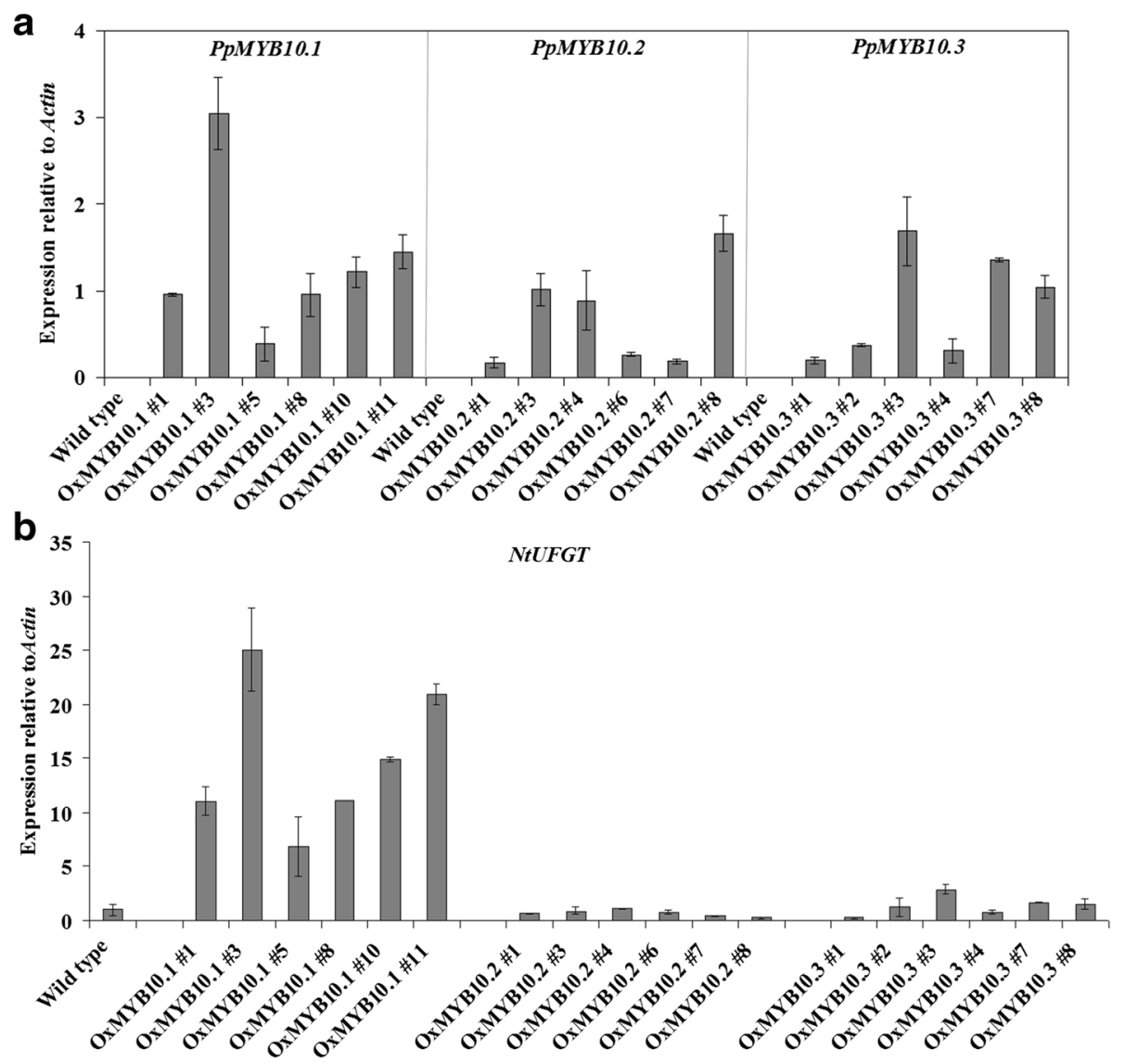

Fig. 5 Expression levels of PPMYB10.1/2/3 transgenes (a) and NtUFGT (b) in transgenic tobacco flowers. Height of the bars and error bars shows the mean and standard error, respectively, from three independent measurements

'Mochizuki' and 'Akatsuki' (Fig. 6c). 'Akatsuki' flowers showed higher PpUFGT expression levels than 'Mochizuki' flowers, leading to higher total anthocyanin content in 'Akatsuki' flowers (Fig. 6a, b). It was correlated with the expression of PpMYB10.1 in flowers of 'Mochizuki' and 'Akatsuki'. PpMYB10.2 transcription was high but not associated with anthocyanin content, and only trace PpMYB10.3 expression was detected in flowers of 'Mochizuki' and 'Akatsuki'. These results indicate that PpMYB10.1 is responsible for anthocyanin accumulation in flowers of 'Mochizuki' and 'Akatsuki'. PpMYB10.2 and $P p M Y B 10.3$ have functions other than anthocyanin accumulation in leaves and flowers of 'Mochizuki' and 'Akatsuki'.

\section{Functional analysis of PpMYB10.1 by transient promoter assay in a heterologous system}

To evaluate the regulatory capacity of PpMYB10.1 on the expression of PpUFGT, transient expression of a FLUC reporter gene under the control of the putative PpUFGT promoter regulated by $P$ PMYB10.1 alone or a combination of PpMYB10.1 and PpbHLH3 was evaluated in Nicotiana benthamiana leaves. As shown in Fig. 7, activity of the PpUFGT promoter was significantly induced by $P p M Y B 10.1$ in the presence of PpbHLH3. $P p M Y B 10.1$ and PpbHLH3 alone could not significantly increase the promoter activity of PpUFGT.

\section{Investigation of the differences in PpMYB10.1 expression in 'Mochizuki' and 'Akatsuki'}

Since PpMYB10.1 was differentially expressed in redskinned 'Akatsuki' and white-skinned 'Mochizuki', we then intended to investigate the expression of $N A C$ including Blood $(P p B L)$ and SQUAMOSA promoterbinding protein-like transcription factor (PpSPL1) genes that have been reported as upstream transcription factors for $M Y B$ regulation in red-fleshed peach [27]. The expression level of $P p B L$ in 'Akatsuki' was higher than in 'Mochizuki', but apparent expression of $P p B L$ was also recorded even in white-skinned 'Mochizuki' albeit less much (Additional file 3: Figure S3). Expression of PpSPL1, which was believed as a transcriptional repressor of the promoter of $P p M Y B 10.1$ [27], was inversely correlated with the transcription of $P p M Y B 10.1$ in fruit skin during fruit development of 'Mochizuki' and 'Akatsuki'. These results indicated that PpMYB10.1 expression 

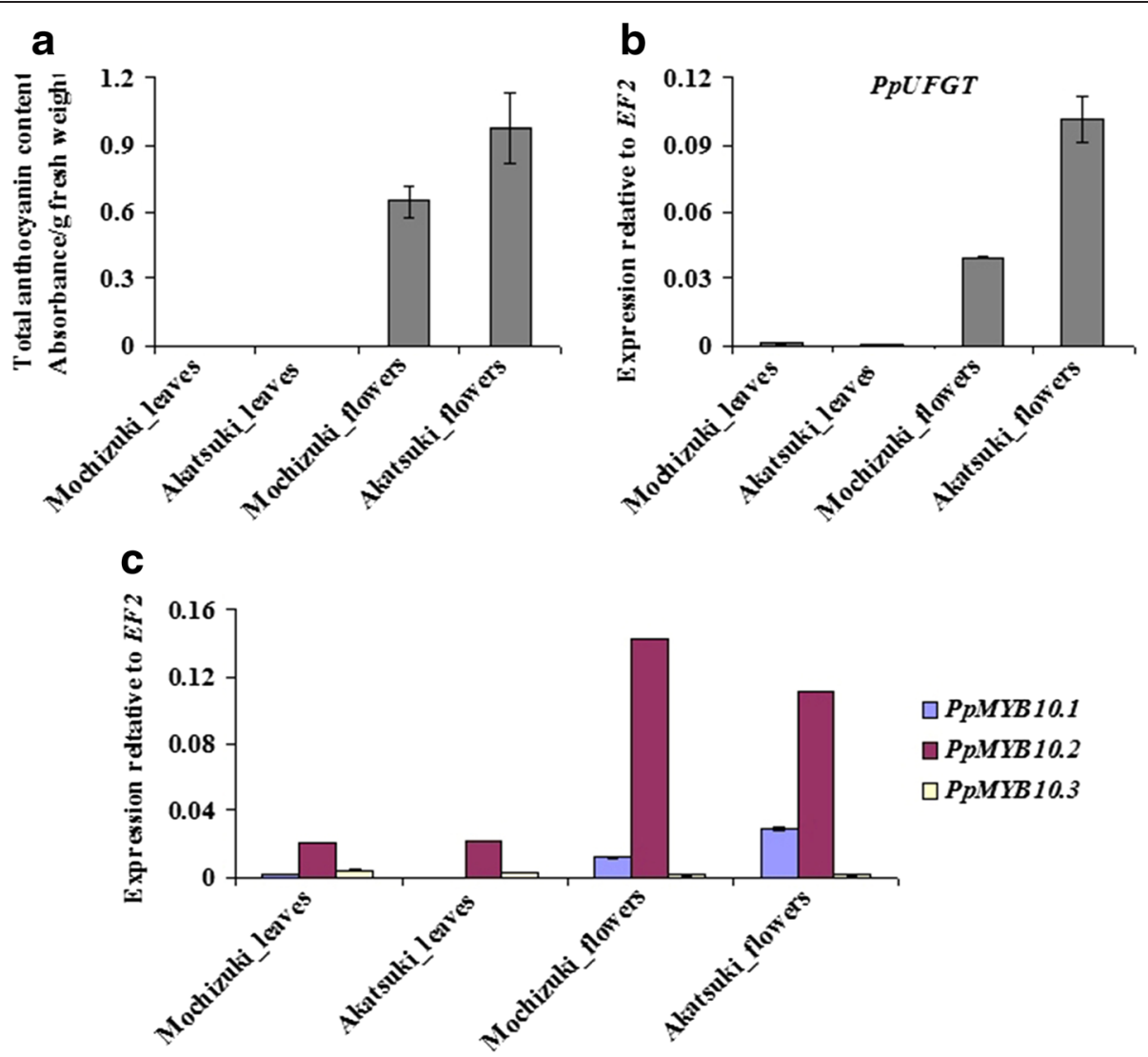

Fig. 6 a Total anthocyanin content. Expression levels of PpUFGT (b) and PPMYB10.1/2/3 (c) in leaves and flowers of 'Mochizuki' and 'Akatsuki'. Height of bars and error bars shows the mean and standard error, respectively, from three independent measurements

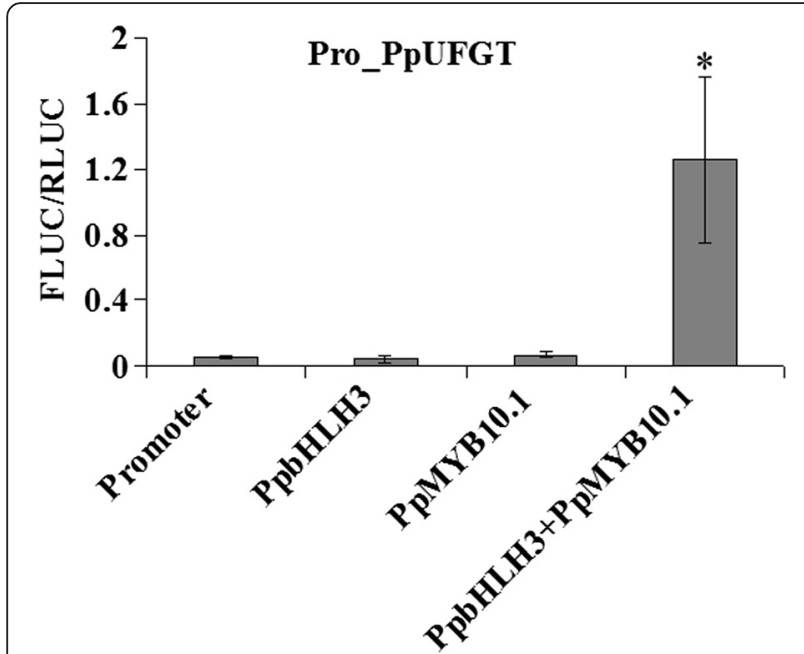

Fig. 7 Transient activation of the 2000-bp upstream regions of PpUFGT by PpMYB10.1 alone or in combination with PpbHLH3. Height of the bars and error bars shows the mean and standard error, respectively, from six independent measurements. The asterisk indicates a significant difference $(P<0.05)$ from leaves infiltrated with the only promoter of P PUFGT, by using the two-tailed Student's t-test and red skin color was regulated by $P p B L$ and PpSPL1 as in the case of blood flesh [27]. However, it was noticed that $P p B L$ expression was increased in skin of stage 5 of 'Mochizuki' fruits where PpMYB10.1 expression level remained low, indicating other factors such as genomic structure may be also involved in the regulation of PpMYB10.1 activity. Therefore, we further investigated the genomic sequences of $P p M Y B 10.1$ in redskinned cultivar and white-skinned cultivar. There were many insertion/deletion (InDel) and single nucleotide polymorphisms (SNPs) in the sequences of up- and down-streams as well as introns in Mochizuki-type PpMYB10.2 compared to Akatsuki-type PpMYB10.1 (tentatively designated as MYB10.1-1 for Akatsuki-type and MYB10.1-2 for Mochizuki-type, respectively) (Fig. 8). Especially, the coding regions of MYB10.1-2 type showed one deletion at 8-13 nt of exon I and three SNPs at 431, 464 and 617 nt of exon III, causing two amino acid deletion and four amino acid substitution in coding regions of MYB10.1-2 compared to that of MYB10.1-1 type (Additional file 4: Figure S4). Based on these three SNPs of exon III, we investigated the mRNA sequences of PpMYB10.1 in 'Shimizu-hakuto', which was preliminary found to be heterozygous for PpMYB10.1 


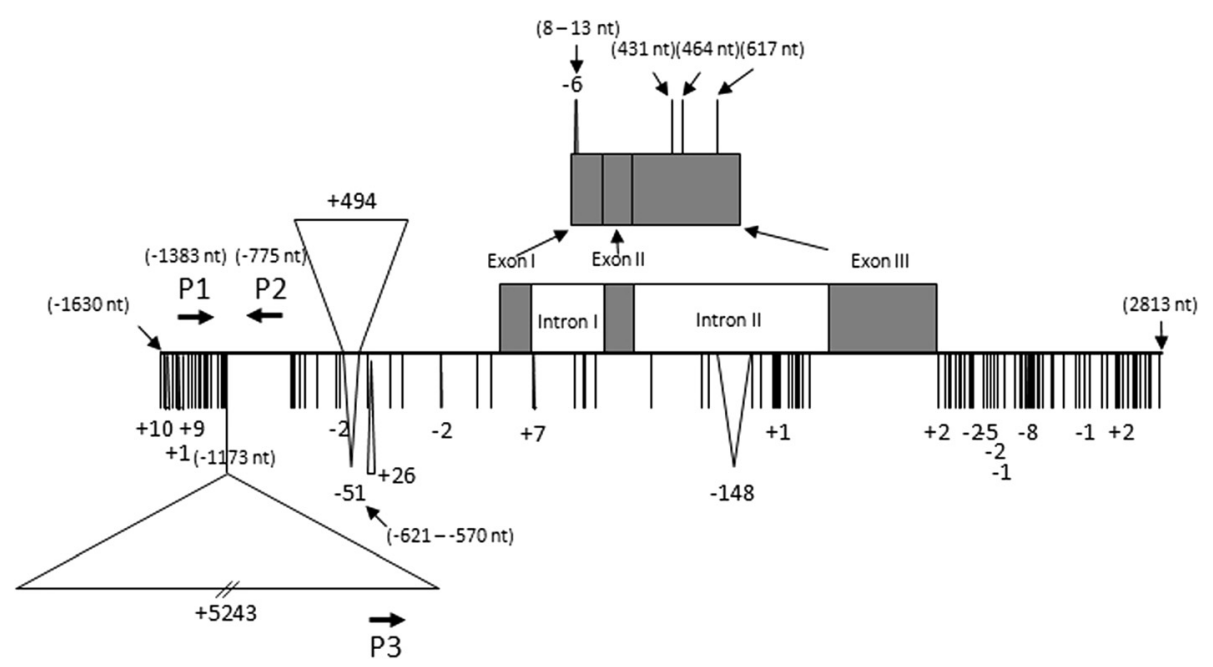

Fig. 8 Genomic structure of PpMYB10.1 allele. "I", "+n", and "-n" indicate the single nucleotide polymorphism, number of nucleotide insertion, and number of nucleotide deletion, respectively, of MYB10.1-2 type compared to MYB10.1-1 type. (n nt) indicate the nucleotide position relative to start codon ATG. P1, P2, and P3 are three primers used to discriminate MYB10.1 allelic types

locus (MYB10.1-1/MYB10.1-2). The results showed that all the transcripts of PpMYB10.1 from 60 independent clones were derived from MYB10.1-1 only (Table 1), indicating that the reason for induction of $P p M Y B 10.1$ expression is ascribed to $P p M Y B 10.1$ genomic structure. Based on the differences in two PpMYB10.1 types, we tried to classify 23 Japanese peach cultivars. Thirteen cultivars showing red skin color (color index $=2$, Additional file 5: Figure S5a) including 'Akatsuki' possess two MYB10.1-1 type, while those with white skin color (color index $=0$, Additional file 5: Figure S5b) including 'Mochizuki' had two MYB10.1-2 type (Table 2, Additional file 6: Figure S6). Among seven analyzed cultivars with pale red skin color (color index $=1$, Additional file 5: Figure S5c), three cultivars had two MYB10.1-1 type, while other four cultivars were consisted of one MYB10.1-1 type and one MYB10.2 type (Table 2, Additional file 6: Figure S6). Although we cannot exclude other possibilities such as epigenetic regulation for the differences in PpMYB10.1 expression in 'Mochizuki' and 'Akatsuki', these results demonstrated that the skin color phenotypes can be partially explained by the differences in PpMYB10.1 allelic types.

Table 1 Sequence analysis of PpMYB10.1 transcript in PpMYB10.1-heterozygous 'Shimizu-hakuto'

\begin{tabular}{lllll}
\hline Type & SNPs & & & $\begin{array}{l}\text { Number } \\
\text { of CDNA } \\
\text { clone }\end{array}$ \\
\hline MY1 nt & $464 \mathrm{nt}$ & $617 \mathrm{nt}$ & \\
MYB10.1-1 & $\mathrm{C}$ & $\mathrm{C}$ & $\mathrm{C}$ & 0 \\
\hline
\end{tabular}

\section{Discussion}

PpMYB10.1 contributes to anthocyanin accumulation in peach fruit skin and flowers

The transcription factor MYB10 group has been reported to be responsible for the red coloration in the skin of several Rosaceae fruit trees such as apple, pear, and strawberry [21, 23, 28]. For peach, Ravaglia et al. [25] first demonstrated the importance of PpMYB10 (corresponding to PpMYB10.2) in anthocyanin accumulation in nectarine, while Rahim et al. [26] reported that expression levels of PpMYB10.1 and PpMYB10.3 correlated with the anthocyanin content of the peel, mesocarp, and mesocarp around the stone. These results were not consistent with our expression analysis results that PpMYB10.2/3 transcript was very low in the redskinned samples and only expression of PpMYB10.1 was highly consistent with anthocyanin accumulation throughout fruit development (Figs. 2b and 3b). Recently, Zhou et al. [27] also showed that only PpMYB10.1 expression is correlated with anthocyanin level in blood-flesh peach. We assumed that the anthocyanin biosynthetic mechanism may depend on the analyzed peach cultivars and fruit tissues. In this study, the direct contribution of PpMYB10.1 to anthocyanin accumulation and red coloration of fruit skin and flowers of 'Akatsuki' was found (Figs. 2b, 3b and 6). During fruit development in 'Mochizuki' and 'Akatsuki', PpMYB10.1 expression was highly correlated with PpUFGT (Fig. 2c). Transient expression analysis of tobacco leaves indicated that PpMYB10.1/ PpbHLH3 significantly increased the activities of the PpUFGT promoter (Fig. 7). Furthermore, overexpression of PpMYB10.1 resulted in higher anthocyanin content and 
Table 2 Classification of 23 Japanese peach cultivars according to red color index and PpMYB10.1 allele

\begin{tabular}{llr}
\hline Color index & Cultivar & PpMYB10.1 allele \\
\hline 0 & Mochizuki, Hanashimizu, and Yamate-shimizu & MYB10.1-2/MYB10.1-2 \\
1 & Shimizu-hakuto, Yamato-hakuto, Shimizu-hakuto RS, and Hakushu & MYB10.1-1/MYB10.1-2 \\
& Hakuto, Setouchi-hakuto, and Sakigake-hakuto & MYB10.1-1/MYB10.1-1 \\
2 & Akatsuki, Hakuho, Hanayome, & MYB10.1-1/MYB10.1-1 \\
& Hikawa-hakuho, Kanouiwa-hakuto, Kawanakajima-hakuto, Hakurei, Natsugokoro, Misakakko, Benishimizu, \\
& Asama-hakuto, Chikusa-hakuto, and Tenshin suimitsu & \\
\hline
\end{tabular}

deeper red color in transgenic tobacco and increased only NtUFGT expression but no other branching genes in the flavonoid biosynthetic pathway (Fig. 5b, Additional file 2: Figure S2). In addition, PpMYB10.1 alone contributes to anthocyanin accumulation and red coloration in the flesh, like in the skin of 'Akatsuki' fruit (Additional file 7: Figure S7). These results confirm that PpMYB10.1 is the key regulator of anthocyanin biosynthesis and that it successfully activates the promoter of PpUFGT at least in Japanese peach cultivars, including 'Akatsuki'.

\section{PpMYB10.2/3 contributes to processes other than anthocyanin accumulation}

Expression levels of $P p M Y B 10.2 / 3$ were low throughout the fruit development of 'Mochizuki' and 'Akatsuki' and were not associated with anthocyanin accumulation and red coloration (Fig. 3b). A considerable level of PpMYB10.2 expression was detected in peach leaves that do not contain anthocyanin, and high expression of $P p M Y B 10.2$ was not also consistent with the anthocyanin content of flowers of 'Mochizuki' and 'Akatsuki' (Fig. 6). Rahim et al. [26] showed that PpMYB10.2 contributes to anthocyanin accumulation during leaf senescence and flower development. However, a recent study indicated that PpMYB10.4 on linkage group 6 regulates anthocyanin accumulation in peach leaf, while PpMYB10.2 do not contribute to the leaf red coloration [29]. In this study, our results also proposed that PpMYB10.2/3 do not play roles in the accumulation of anthocyanin in peach leaves and flowers (Fig. 6). Moreover, introduction of $P p M Y B 10.2 / 3$ did not alter the flavonoid biosynthetic genes in tobacco as well anthocyanin accumulation in transgenic tobacco flowers (Fig. 4, Additional file 2: Figure S2). In Arabidopsis, AtMYB75 (NM_104541) and AtMYB90 (AF062915), named Production of Anthocyanin Pigment 1 (AtPAP1) and 2 (AtPAP2), respectively, were identified as regulators of anthocyanin biosynthesis. AtPAP1 and AtPAP2 share high sequence identity and were clustered together in the MYB phylogeny tree constructed by Ravaglia et al. [25]. However, overexpression of not AtPAP2 but AtPAP1 stimulates expression level of the anthocyanin structural gene and anthocyanin accumulation in seedlings of transgenic Arabidopsis plant [30]. Similarly,
PpMYB10.1/2/3 share high sequence identity at amino acid level. We hypothesized that the R/B-like bHLH binding motifs ([DE] $\left.\mathrm{Lx}_{2}[\mathrm{RK}] \mathrm{x}_{3} \mathrm{Lx}_{6} \mathrm{Lx}_{3} \mathrm{R}\right)$ of $P p M Y B 10.2 / 3$ showed an amino acid different with those of PpMYB10.1 (Fig. 3a) [31], by which $P p M Y B 10.2 / 3$ proteins probably act with different bHLHs with PpMYB10.1 to play roles in processes other than anthocyanin biosynthesis.

Preliminary comparisons of MYB10.1 alleles of white-skinned and red-skinned peach cultivars

Since PpMYB10.1 is a key regulatory gene for anthocyanin accumulation in skin, flower, and flesh, we tried to obtain a preliminary insight into the differences of PpMYB10.1 activation observed in 'Mochizuki' and 'Akatsuki'. We first tried to find the cause in the upstream transcription factors of $P p M Y B 10.1$. Expression analysis of PpSPL1 and PpNAC indicated that these genes could be one of the factors for regulating PpMYB10.1 expression levels in 'Mochizuki' and 'Akatuski', but other factors would be also involved in the cause for differential expressions observed (Fig. 3b). To confirm this, we identified the transcribed PpMYB10.1 types using 'Shimizu-hakuto'. The results clearly showed that all the transcribed $P p M Y B 10.1$ types were derived from not MYB10.1-2 but MYB10.1-1 (Table 1), indicating that the activation of $P p M Y B 10.1$ expression depends on PpMYB10.1 allelic types. We then compared the differences in nucleotide sequences of PpMYB10.1 ORFs between white-skinned 'Mochizuki' and red-skinned 'Akatsuki' peach, causing different PpMYB10.1 proteins were translated from MYB10.1-1 and MYB10.1-2 types (Additional file 4: Figure S4). Moreover, several sequence differences in MYB10.1-1 and MYB10.1-2 including InDels and SNPs were recorded in the $5^{\prime}$-upstream, $33^{\prime}$-downstream, and intron (Fig. 8). Thus, genomic sequences of PpMYB10.1 using 23 Japanese peach cultivars with being different skin color enabled to classify into two types of PpMYB10.1, MYB10.1-1 and MYB10.1-2 type, where two MYB10.1-2 types resulted in the white skin color, while two MYB10.1-1 or MYB10.1-1/MYB10.1-2 types showed red or pale red skin color (Table 2). The question that which sequence differences among MYB10.1-1 and MYB10.1-2 types are the cause for activation and inactivation of PpMYB10.1 was raised. Zhou et al. [27] who 
surveyed the differences in DNA sequences of PpMYB10.1 between Chinese white-fleshed and red-fleshed peach cultivars, reported a 483-bp insertion and a SNP in the promoter region and the second intron of 'Dahongpao' blood-fleshed cultivar, respectively. This 483-bp insertion seems to be comparable to the 494-bp InDel found in our study (Fig. 8). However, this 483-bp insertion was not proved to link to the red flesh color in other Chinese peach cultivars [27]; in other word, this insertion was not a cause for activation/inactivation of PpMYB10.1. Similarly, considering that peach reference MYB10.1 gene using red peach cultivar 'Lovell' possessed this insertion (see https://www.rosaceae.org/species/prunus/prunus_persica), we also assumed that 494-bp insertion in MYB10.1-2 did not relate to the function, but our 494-bp InDel was found to associate with the red color index in 23 Japanese cultivars. Twenty-three Japanese peach cultivars examined in this study share close genetic relationships to each other as you can see the several sports of 'Hakuto' peach (Table 2), which may explain why PpMYB10.1 allelic types were associated with the red color indexes in skin of these cultivars.. Therefore, we are not sure if our results can apply to other peach cultivars/ strains especially other than Japanese cultivars. By contrast, we also found other InDels and many SNPs in the 5 '-upstream, 3'-downstream, exons and introns in PpMYB10.1 alleles of Japanese peach cultivars. It suggested that the genomic structures of Japanese peach cultivars are not completely similar with those of Chinese peach cultivars [27]. At present, we have not yet determined the actual key sequence differences in MYB10.1-1 and MYB10.1-2 types that lead to the activation of MYB10.1-1 type. Further surveys are required to establish the essential factors in DNA sequences that directly contributed to the expression of PpMYB10.1 in red-skinned peach.

\section{Conclusions}

We concluded that PpMYB10.1 is a major regulator of anthocyanin accumulation in red-skinned peach and that a close relationships between PpMYB10.1 allelic type and skin color phenotype (at least in 23 Japanese cultivars). This finding will enable further investigation of DNA sequences of $P p M Y B 10.1$, providing essential information to establish DNA markers linked to white- and red-skinned peach for fruit breeders.

\section{Methods}

\section{Plant materials}

Peach fruit were collected from 'Mochizuki' and 'Akatsuki' trees grown in the orchard of the NARO Institute of Fruit Tree Science, Tsukuba, Japan (latitude $36^{\circ} \mathrm{N}$, longitude $140^{\circ} \mathrm{E}$ ). Leaves and flowers were collected on April 26, 2013. Peach skin and flesh were separated manually from three to nine fruit at five different stages of fruit development (Additional file 8: Figure S8). Other cultivars shown in Table 2 were cultivated in the orchard of the Okayama Prefectural Technology Center for Agriculture, Forestry, and Fisheries, Okayama, Japan (latitude $35^{\circ} \mathrm{N}$, longitude $134^{\circ} \mathrm{E}$ ). The skin samples were frozen in liquid nitrogen and stored at $-80{ }^{\circ} \mathrm{C}$ until analysis. We classified 23 peach cultivars based on the degree/intensity of red pigmentation in the peach skin; we defined color index 0,1 and 2 as white skin color, pale red skin color and red skin color, respectively (see Additional file 5: Figure S5).

\section{qRT-PCR and expression analysis}

The peach samples were ground in a mortar with liquid nitrogen, and total RNA was extracted from the frozen powder by using the hot-borate method [32]. Gel electrophoresis and spectrophotometry were performed to test the quality and concentration of the extracted RNA, respectively. For first-strand cDNA synthesis, $1 \mu \mathrm{g}$ of high-quality total RNA was used for reverse transcription with the SuperScript VILO cDNA Synthesis Kit (Invitrogen, Carlsbad, CA, USA). A 20-fold dilution of the 20- $\mu \mathrm{l}$ cDNA was used as the template for qRT-PCR. Target genes were shown in Additional file 9: Table S1. RT-PCR primers were designed using the Primer3 website (http://bioinfo.ut.ee/primer3-0.4.0/primer3/, Additional file 9: Table S1). RT-PCR products were confirmed by fragment sizes, melting curves, and sequencing. Expression levels of target genes were analyzed by relative quantification, with the elongation factor 2 housekeeping gene (EF2, ppa001368m) as the reference gene. The reaction mixture $(10 \mu \mathrm{l})$ contained $1 \mu \mathrm{l}$ of diluted cDNA sample, $0.5 \mu \mathrm{l}$ of each primer $(10 \mu \mathrm{M}), 5 \mu \mathrm{l}$ of GeneAce SYBR qPCR Mix $\alpha$ Low ROX (Nippon Gene, Tokyo, Japan), and DEPC water. qRT-PCR was performed using the 7500 Real Time PCR System (Applied Biosystems, Foster City, CA, USA), and the results were analyzed with the 7500 System Sequence Detection Software ver. 1.4. Three biological replications with three technical replications for each sample were used for real-time analyses.

\section{Plasmid vector construction for transformation}

ORFs of PpMYB10.1/2/3 were generated from first-strand cDNA of 'Akatsuki' by using PCR with KOD-Neo DNA polymerase (Toyobo, Osaka, OSK, Japan). Purified PCR fragments with the expected size bands were cloned into the pENTR/D-TOPO vector (Invitrogen), according to the manufacturer's instructions. Subsequently, the ORFs of PpMYB10.1/2/3 were inserted into the pGWB2 vector under transcriptional control of the $35 \mathrm{~S}$ CaMV promoter [33] by using LR clonase (Invitrogen). Recombinant pGWB2 plasmids were then transferred into Agrobacterium tumefaciens LBA4404 by using the heat-shock 
method and grown at $28{ }^{\circ} \mathrm{C}$ on Luria-Bertani medium with kanamycin, hygromycin, and rifampicin.

\section{Stable transformation of $N$. tabacum}

Excised leaves of N. tabacum SR1 from 4-week-old seedlings were used as the explant material for co-cultivation with A. tumefaciens LBA4404 harboring PpMYB10.1/2/3 overexpression constructs. The transformation was carried out as described previously by Dobhal et al. [34]. Flowers of 3-month-old transgenic tobacco plants were collected and immediately frozen in liquid nitrogen and then stored at $-80{ }^{\circ} \mathrm{C}$ for RNA isolation and anthocyanin analysis. Total RNA was extracted from the transgenic tobacco flowers by using cetyltrimethylammonium bromide buffer and a silica column-based extraction method [35]. Expression levels of NtFLS (AB289451), NtANR (AM791704), NtLAR (AM827419), NtUFGT (GQ395697), and transgenes $P p M Y B 10.1 / 2 / 3$ were analyzed using NtActin (X69885) as the reference. Primers used for gene expression analysis of transgenic tobacco are listed in Additional file 9: Table S1.

\section{Transient dual-luciferase assay}

The putative promoter region of peach PpUFGT, which is $2000 \mathrm{bp}$ upstream from the start codon, was amplified using genomic DNA extracted from "Akatsuki" fruit (primers are listed in Additional file 9: Table S1). The promoter region of PpUFGT was cloned into pGWB35 [33], in which the firefly luciferase (FLUC) gene is under the control of the cloned promoters. As the internal standard vector, renilla luciferase (RLUC) gene amplified from the pRL-SV40 vector (Promega, Madison, WI, USA) was cloned into the pBGW2 vector. ORFs of PpMYB10.1 and PpbHLH3 (ppa002884m) amplified from "Akatsuki" first-strand cDNA were also cloned into pGWB2. All resultant plasmids were transformed into $A$. tumefaciens LBA4404. Transient expression assays were performed using $N$. benthamiana leaves with the method available in our laboratory [36]. FLUC and RLUC were assayed using dual luciferase assay reagents (Promega, Madison, WI, USA), and their activities were detected with the ChemiDoc ${ }^{\text {Tm }}$ MP System (Bio Rad, Hercules, CA, USA). Data were collected as the ratio of FLUC to RLUC. Six independent biological replications (six leaves from different plants) and technical replications (2-3 injections of the same leaf) were performed for each treatment.

\section{Measurements of total anthocyanin}

The peach and transgenic tobacco samples were placed in $10 \mathrm{ml}$ of methanol:hydrochloric acid $(99: 1, \mathrm{v} / \mathrm{v})$. Extractions were performed overnight in the dark at $4{ }^{\circ} \mathrm{C}$. Absorbance of each extract was measured at 530, 620 and $650 \mathrm{~nm}$ by using a spectrophotometer (UV-2450; Shimadzu, Kyoto, Japan). Relative anthocyanin content was normalized using the following formula: Normalized OD530 $=[($ OD530-OD650 $)-0.2 \times($ OD650-OD620) $]$ [37 $]$. Three independent biological replicates were performed for each measurement.

\section{Analysis of PpMYB10.1 alleles transcribed in 'Shimizu-hakuto'} skin

The skin of 'Shimizu-hakuto' whose PpMYB10.1 allelic types were preliminary analyzed as MYB10.1-1 and MYB10.1-2 types, were frozen by liquid nitrogen and subsequently powdered using a Multi Beads Shocker (Yasui Kikai, Osaka, Japan). Total RNA was extracted from powdered skin with cetyltrimethylammonium bromide method [38]. For first-strand cDNA synthesis, $1 \mu \mathrm{g}$ of high-quality total RNA was used for reverse transcription with the SuperScript VILO cDNA Synthesis Kit (Invitrogen, Carlsbad, CA, USA). RT-PCR primers for discriminating PpMYB10.1 allelic types were shown in Additional file 9: Table S1. RTPCR products were subcloned into Zero Blunt TOPO PCR cloning kit (Invitrogen), and sequencing.

\section{Analysis of genomic sequences of PpMYB10.1 in 23 peach} cultivars

We then determined the genomic sequences using 23 Japanese peach cultivars (Table 2). Genomic DNA from peach skin was extracted using the DNeasy Plant Mini Kit (Qiagen, Tokyo, Japan). We first carried out PCR using genomic DNA with the primers for discriminating the InDel using P1, P2, and P3 primer sets (primers in Additional file 9: Table S1). Briefly, PCR was carried out with the mixtures of 3 primers, where the band of either 609 or 426 bp was amplified in case of MYB10.1-1/MYB10.1-1 (by P1/P2) or MYB10.1-2/MYB10.1-2 (P2/P3), respectively, while both bands were amplified for MYB10.1-1/MYB10.1-2. Here, the combination of $\mathrm{P} 1 / \mathrm{P} 2$ primer could not generate band in MYB10.1-2/MYB10.1-2 types due to the long size (over $5800 \mathrm{bp}$ ) of amplicon. Then, the sequences in the $5^{\prime}$-upstream, $3^{\prime}$-downstream, exon and intron were obtained by PCR using several primer sets (data not shown). PCR products were subcloned and sequenced as aforementioned.

\section{Availability of supporting data}

All supporting data are included as additional files.

\section{Additional files}

Additional file 1: Figure S1. (a) Confirmation of the presence of transgenes PpMYB10.1/2/3 in the transgenic tobacco genome by using the CaMV 35 S primer (5'-TCCACTGACGTAAGGGATGAC-3') and PpMYB10.1/ 2/3 ORF reverse primers. POS, positive control; WT, wild-type tobacco plant; $\#$ n, independent line numbers. (b) Red coloration in capsule skin of transgenic tobacco plants overexpressing PpMYB10.1. (JPG 2499 kb)

Additional file 2: Figure S2. Expression levels of branching genes involved in the flavonoid biosynthetic pathway - NtFLS, NtLAR, and 
NtANR in transgenic tobacco flowers. Height of the bars and error bars shows the mean and standard error, respectively, from three independent measurements. (JPG $646 \mathrm{~kb}$ )

Additional file 3: Figure S3. Expression levels of $P p B L$ and $P p S P L 1$ in the skin of 'Mochizuki' and 'Akatsuki' during fruit development. Height of bars and error bars shows the mean and standard error, respectively, from three independent measurements. (JPG $49 \mathrm{~kb}$ )

Additional file 4: Figure S4. Amino acid alignments of MYB10.1-1 and MYB10.1-2 ORFs amplified from first-strand cDNA of 'Shimizu-hakuto'. (JPG $83 \mathrm{~kb}$ )

Additional file 5: Figure S5. Photographs of peach fruits classified to red color index 0 (a) 'Hanashimizu', 1 (b) 'Shimizu-hakuto', and 2 (c) 'Hakuho'. (JPG 1479 kb)

Additional file 6: Figure S6. Analysis of MYB10.1 alleles in 23 Japanese peach cultivars using P1, P2, and P3 primers. 'Akatsuki', 'Hakuho', 'Hanayome', 'Hikawa-hakuho', 'Kanouiwa-hakuto', 'Kawanakajima-hakuto', 'Hakurei', 'Natsugokoro', 'Misakakko', 'Benishimizu', 'Asama-hakuto', 'Chikusa-hakuto', 'Tenshin suimitsu', 'Hakuto', 'Setouchi-hakuto', and 'Sakigake-hakuto' showed 609-bp bands (MYB10.1-1/MYB10.1-1). 'Mochizuki', 'Hanashimizu', and 'Yamate-shimizu' showed 426-bp bands (MYB10.12/MYB10.1-2). 'Shimizu-hakuto',

'Yamato-hakuto', 'Shimizu-hakuto RS', and 'Hakushu' had both bands (MYB10.1-1/MYB10.1-2). (JPG 108 kb)

Additional file 7: Figure S7. 'Akatsuki' usually shows white flesh, but can accumulate anthocyanin to some extent depending on the ripening stages and environmental conditions, which are not yet fully addressed. In contrast, 'Mochizuki' seldom shows red pigmentation in its flesh. (a) Photographs of fruit flesh. (b) Total anthocyanin content. (c) Expression levels of PpMYB10.1/2/3 in the flesh of 'Mochizuki' and 'Akatsuki' during fruit development. Height of bars and error bars shows the mean and standard error, respectively, from three independent measurements. (JPG $751 \mathrm{~kb}$ )

Additional file 8: Figure S8. Weights (a) and sizes of equatorial diameters (b) of 'Mochizuki' and 'Akatsuki' fruit harvested on May 17 (S1), June 03 (S2), June 27 (S3), July 10 (S4), and July 24 (S5), 2013. (JPG 901 kb)

Additional file 9: Table S1. Primers used in this study. (DOCX 28 kb)

\section{Abbreviations}

ANS: anthocyanidin synthase; bHLH: basic helix-loop-helix; BL: blood; $\mathrm{CHI}$ : chalcone isomerase; CHS: chalcone synthase; DFR: dihydroflavonol-4reductase; F3H: flavanone 3-hydroxylase; FLUC: firefly luciferase; ORF: open reading frame; qRT_PCR: quantitative real-time polymerase chain reaction; RLUC: renilla luciferase; SPL1: SQUAMOSA promoter-binding protein-like transcription factor; UFGT: UDP-glucose:flavonoid-3-O-glucosyltransferase.

\section{Competing interests}

The authors declare that they have no competing interests.

\section{Authors' contributions}

TM and KO designed the study. PAT, SB, and HY performed the experiments. $\Pi \mathrm{T}$ and $\mathrm{SH}$ contributed to the color index analysis of peach fruits. PAT and TM analyzed the data and wrote the paper. All authors read and approved the final manuscript.

\section{Acknowledgements}

This study was supported by Grants-in-Aid for Scientific Research (no. 25.03516) from the Japan Society for the Promotion of Science (JSPS). We appreciate Dr. T Imai for his supply of tobacco SR1 plants.

\section{Author details}

'NARO Institute of Fruit Tree Science, 2-1 Fujimoto, Tsukuba, Ibaraki 305-8605, Japan. ${ }^{2}$ Research Institute for Agriculture, Okayama Prefectural Technology Center for Agriculture, Forestry, and Fisheries, 1174-1 Koda-Oki, Akaiwa, Okayama 709-0801, Japan. ${ }^{3}$ Research Institute for Biological Sciences, Okayama Prefectural Technology Center for Agriculture Forestry, and Fisheries, 7549-1 Yoshikawa, Kibi-chou, Okayama 716-1241, Japan.
Received: 9 September 2015 Accepted: 4 November 2015

Published online: 18 November 2015

\section{References}

1. Tsuda T, Yamaguchi M, Honda C, Moriguchi T. Expression of anthocyanin biosynthetic genes in the skin of peach and nectarine fruit. J Amer Soc Hort Sci. 2004;129(6):857-62.

2. Brandi F, Bar E, Mourgues F, Horváth G, Turcsi E, Giuliano G, et al. Study of 'Redhaven' and its white-fleshed mutant suggests a key role of CCD4 carotenoid dioxygenase in carotenoid and norisoprenoid volatile metabolism. BMC Plant Biol. 2011;11:24.

3. Connors $\mathrm{CH}$. Some notes on the inheritance of unit characters in the peach. Proc Amer Soc Hort Sci. 1920;16:24-36.

4. Bailey JS, French HP. The inheritance of certain fruit and foliage characters in peach. Mass Agr Expt Sta Bul. 1949;452:2-31.

5. Adami M, Franceschi PD, Brandi F, Liverani A, Giovannini D, Rosati C, et al. Identifying a carotenoid cleavage dioxygenase (ccd4) gene controlling yellow/white fruit flesh color of peach. Plant Mol Biol Rep. 2013;31:1166-75.

6. Falchi R, Vendramin E, Zanon L, Scalabrin S, Cipriani G, Verde I, et al. Three distinct mutational mechanisms acting on a single gene underpin the origin of yellow flesh in peach. Plant J. 2013;76(2):175-87.

7. Fukamatsu Y, Tamura T, Hihara S, Oda K. Mutations in the CCD4 carotenoid cleavage dioxygenase gene of yellow-flesh peaches. Biosci Biotechnol Biochem. 2013;77(12):2514-6.

8. Bai S, Tuan PA, Tatsuki M, Yaegaki H, Ohmiya A, Yamamizo C et al. Knockdown of carotenoid cleavage doxygenase 4 (CCD4) via virus-induced gene silencing confers yellow coloration in peach fruit: evaluation of gene function related to fruit traits. Plant Mol Biol Rep. 2015. (In Press, doi:10.1007/s11105-015-0920-8).

9. Ma J, Li J, Zhao J, Zhou H, Ren F, Wang L, et al. Inactivation of a gene encoding carotenoid cleavage dioxygenase (CCD4) leads to carotenoidbased yellow coloration of fruit flesh and leaf midvein in peach. Plant Mol Biol Rep. 2014;32(1):246-57.

10. Jia $\mathrm{H}-\mathrm{J}$, Araki A, Okamoto $\mathrm{G}$. Influence of fruit bagging on aroma volatiles and skin coloration of 'Hakuho' peach (Prunus persica Batsch). Postharvest Biol Technol. 2005;35(1):61-8.

11. Lin-Wang K, Micheletti D, Palmer J, Volz R, Lozano L, Espley R, et al. High temperature reduces apple fruit colour via modulation of the anthocyanin regulatory complex. Plant Cell Environ. 2011;34(7):1176-90.

12. Yu B, Zhang D, Huang C, Qian M, Zheng $X$, Teng $Y$, et al. Isolation of anthocyanin biosynthetic genes in red Chinese sand pear (Pyrus pyrifolia Nakai) and their expression as affected by organ/tissue, cultivar, bagging and fruit side. Sci Hort. 2012;136:29-37.

13. Jaakola L. New insights into the regulation of anthocyanin biosynthesis in fruits. Trends Plant Sci. 2013;18(9):477-83.

14. Kayesh E, Shangguan L, Korir N, Sun X, Bilkish N, Zhang Y, et al. Fruit skin color and the role of anthocyanin. Acta Physiol Plant. 2013;35(10):2879-90.

15. Lin-Wang K, Bolitho K, Grafton K, Kortstee A, Karunairetnam S, McGhie TK, et al. An R2R3 MYB transcription factor associated with regulation of the anthocyanin biosynthetic pathway in Rosaceae. BMC Plant Biol. 2010;10:50.

16. Allan AC, Hellens RP, Laing WA. MYB transcription factors that colour our fruit. Trends Plant Sci. 2008;13(3):99-102.

17. Petroni $\mathrm{K}$, Tonelli C. Recent advances on the regulation of anthocyanin synthesis in reproductive organs. Plant Sci. 2011;181(3):219-29.

18. Koyama K, Numata M, Nakajima I, Goto-Yamamoto N, Matsumura H, Tanaka N. Functional characterization of a new grapevine MYB transcription factor and regulation of proanthocyanidin biosynthesis in grapes. J Exp Bot. 2014;65(15):4433-49.

19. Kobayashi S, Ishimaru M, Hiraoka K, Honda C. Myb-related genes of the Kyoho grape (Vitis labruscana) regulate anthocyanin biosynthesis. Planta. 2002;215(6):924-33

20. Ban Y, Honda C, Hatsuyama Y, Igarashi M, Bessho H, Moriguchi T. Isolation and functional analysis of a MYB transcription factor gene that is a key regulator for the development of red coloration in apple skin. Plant Cell Physiol. 2007;48(7):958-70.

21. Chagné D, Lin-Wang K, Espley RV, Volz RK, How NM, Rouse S, et al. An ancient duplication of apple MYB transcription factors is responsible for novel red fruitflesh phenotypes. Plant Physiol. 2013;161(1):225-39.

22. Feng $S$, Wang $Y$, Yang $S, X u Y$, , Chen $X$. Anthocyanin biosynthesis in pears is regulated by a R2R3-MYB transcription factor PyMYB10. Planta. 2010;232(1):245-55. 
23. Wang Z, Meng D, Wang A, Li T, Jiang $S$, Cong $P$, et al. The methylation of the PCMYB10 promoter is associated with green-skinned sport in Max Red Bartlett pear. Plant Physiol. 2013;162(2):885-96.

24. Dirlewanger E, Graziano E, Joobeur T, Garriga-Calderé F, Cosson P, Howad W et al. Comparative mapping and marker-assisted selection in Rosaceae fruit crops. Proc Natl Acad Sci U S A. 2004;101 (26):9891-6.

25. Ravaglia D, Espley RV, Henry-Kirkm RA, Andreotti C, Ziosi V, Hellens RP, et al. Transcriptional regulation of flavonoid biosynthesis in nectarine (Prunus persica) by a set of R2R3 MYB transcription factors. BMC Plant Biol. 2013;13:68.

26. Rahim MA, Busatto N, Trainotti L. Regulation of anthocyanin biosynthesis in peach fruits. Planta. 2014;240(5):913-29.

27. Zhou H, Lin-Wang K, Wang H, Gu C, Dare AP, Espley RV, et al. Molecular genetics of blood-fleshed peach reveals activation of anthocyanin biosynthesis by NAC transcription factors. Plant J. 2015;82(1):105-21.

28. Lin-Wang K, McGhie T, Wang M, Liu Y, Warren B, Storey R, et al. Engineering the anthocyanin regulatory complex of strawberry (Fragaria vesca). Front Plant Sci. 2014;5:651.

29. Zhou Y, Zhou H, Lin-Wang K, Vimolmangkang S, Espley R, Wang L, et al. Transcriptome analysis and transient transformation suggest an ancient duplicated MYB transcription factor as a candidate gene for leaf red coloration in peach. BMC Plant Biol. 2014;14(1):388.

30. Cominelli E, Gusmaroli G, Allegra D, Galbiati M, Wade HK, Jenkins Gl, et al. Expression analysis of anthocyanin regulatory genes in response to different light qualities in Arabidopsis thaliana. J Plant Physiol. 2008;165(8):886-94.

31. Zimmermann IM, Heim MA, Weisshaar B, Uhrig JF. Comprehensive identification of Arabidopsis thaliana MYB transcription factors interacting with R/B-like BHLH proteins. Plant J. 2004;40(1):22-34.

32. Wan CY, Wilkins TA. A modified hot borate method significantly enhances the yield of high-quality RNA from cotton (Gossypium hirsutum L.). Anal Biochem. 1994;223(1):7-12.

33. Nakagawa T, Kurose T, Hino T, Tanaka K, Kawamukai M, Niwa Y, et al. Development of series of gateway binary vectors, pGWBs, for realizing efficient construction of fusion genes for plant transformation. J Biosci Bioeng. 2007;104(1):34-41.

34. Dobhal S, Pandey D, Kumar A, Agrawal S. Studies on plant regeneration and transformation efficiency of Agrobacterium mediated transformation using neomycin phosphotransferase II (nptll) and glucuronidase (GUS) as a reporter gene. Afr J Biotechnol. 2010;9(41):6853-9.

35. Henderson D, Hammond J. CKC: isolation of nucleic acids from a diversity of plants using CTAB and silica columns. Mol Biotechnol. 2013;53(2):109-17.

36. Bai S, Saito T, Honda C, Hatsuyama Y, Ito A, Moriguchi T. An apple B-box protein, MdCOL11, is involved in UV-B- and temperature-induced anthocyanin biosynthesis. Planta. 2014;240(5):1051-62.

37. Honda C, Kotoda N, Wada M, Kondo S, Kobayashi S, Soejima J, et al. Anthocyanin biosynthetic genes are coordinately expressed during red coloration in apple skin. Plant Physiol Biochem. 2002;40(11):955-62.

38. Murray MG, Thompson WF. Rapid isolation of high molecular weight plant DNA. Nucl Acids Res. 1980;8(19):4321-6.

\section{Submit your next manuscript to BioMed Central and take full advantage of:}

- Convenient online submission

- Thorough peer review

- No space constraints or color figure charges

- Immediate publication on acceptance

- Inclusion in PubMed, CAS, Scopus and Google Scholar

- Research which is freely available for redistribution

Submit your manuscript at www.biomedcentral.com/submit 